\title{
Paraduodenal Hernia: A Rare Case Report
}

\author{
${ }^{*}$ Dr.( Capt ) Shishir Kumar ${ }^{1}$,Dr.Khushboo ${ }^{2}$ \\ ( Ex MO (Surg) 183 Military hospital) \\ 2 ( Junior resident Pathology, Rajendra Institute Of Medical Sciences,Ranchi, Jharkhand) \\ *Corresponding author: *Dr.( Capt ) Shishir Kumar
}

\begin{abstract}
Paraduodenal hernias are also called mesocolic hernias. Mesocolic hernias are unusual congenital hernias in which small intestine herniates behind mesocolon. They can be discovered incidentally during laparotomy for intestinal obstruction or during radiological investigations. This is a case report of left paraduodenal hernia diagnosed intraoperatively after being operated on in emergency setting for intestinal obstruction. The mouth of the sac was obliterated by suture apposition to the posterior wall. The patient was discharged on day 7 after an uneventful recovery.
\end{abstract}

Keywords: Emergency laparotomy, , Intestinal obstruction, Paraduodenal hernia.

\section{Introduction}

Paraduodenal hernias are rare type of hernias resulting from incomplete rotation of midgut. It may lead to intestinal obstruction or are typically detected incidentally at autopsy or laparotomy. More frequently, paraduodenal hernias are seen on radiological imaging ${ }^{1,2}$. We report a case of an individual who presented with intestinal obstruction and was found to have left paraduodenal hernia on laparotomy.

\section{Case report}

A 32 year old male, serving soldier deployed at a border outpost on eastern border of India was brought to the military hospital in Manipur for investigation of history of intermittent colicky epigastric pain for 6 months, complicated by acute pain and distension of central abdomen with obstipation for 2 days. He reported having suffered from several similar episodes of colicky pain for the last 6 months, but the pain resolved spontaneously each time. On this occasion, his abdominal progressed to be generalized in nature accompanied by distension and vomiting. There was no history of previous abdominal surgery. On physical examination, he was moderately dehydrated with tachycardia ( $100 / \mathrm{min}$ ) and normal blood pressure. There was generalized tenderness, most pronounced in epigastrium.

There was no rebound tenderness. Bowel sounds were absent. Blood analysis showed neutrophilic leukocytosis. X ray Abdomen and urine analysis were normal. The diagnosis of Acute Abdomen secondary to Small intestinal obstruction was made. Informed consent was taken and patient was prepared for emergency laparotomy. After a brief period of resuscitation, laparotomy through midline abdominal incision was performed which revealed that proximal jejunum was within a sac like structure to the left of midline. The sac wall was formed by an anomaly of transverse and descending colon.

The inferior mesenteric vessels were located in the medial edge of the anterior wall of defect, posterior edge being formed by posterior abdominal wall. The intestinal loops were entrapped in the defect because of their anomalous fixation by an adhesive band. The bowel was easily reducible from the sac manually. A small segment of the entrapped jejunum showed ischaemic pallor but regained its colour and peristalsis after warm compresses for about 10 minutes. The peritoneal sac opening was apposed to posterior abdominal wall. The patient had active bowel sounds on second post operative day and was fed orally by third post operative day. Abdominal stitches were removed on $7^{\text {th }}$ post operative day. He was discharged on Day 8 . The patient resumed his military duties, albeit in a restrained manner after 1 month of recuperation at home and has been well since discharge. 


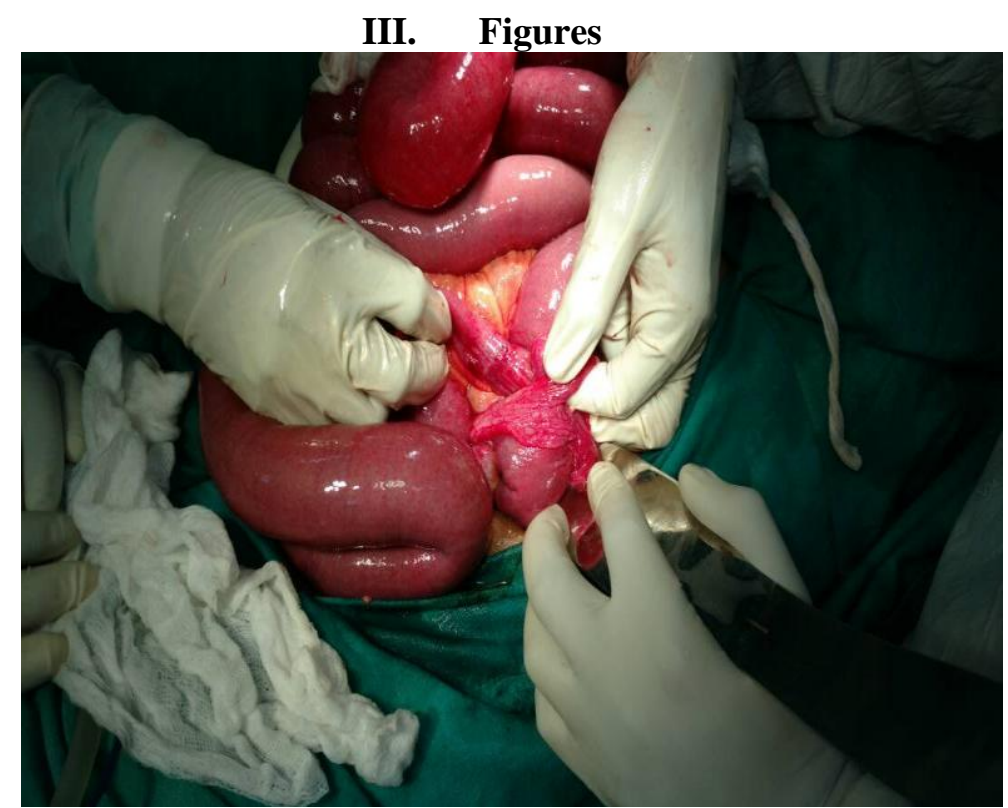

Fig 1: Band trapping the bowel loops inside the peritoneal defect.

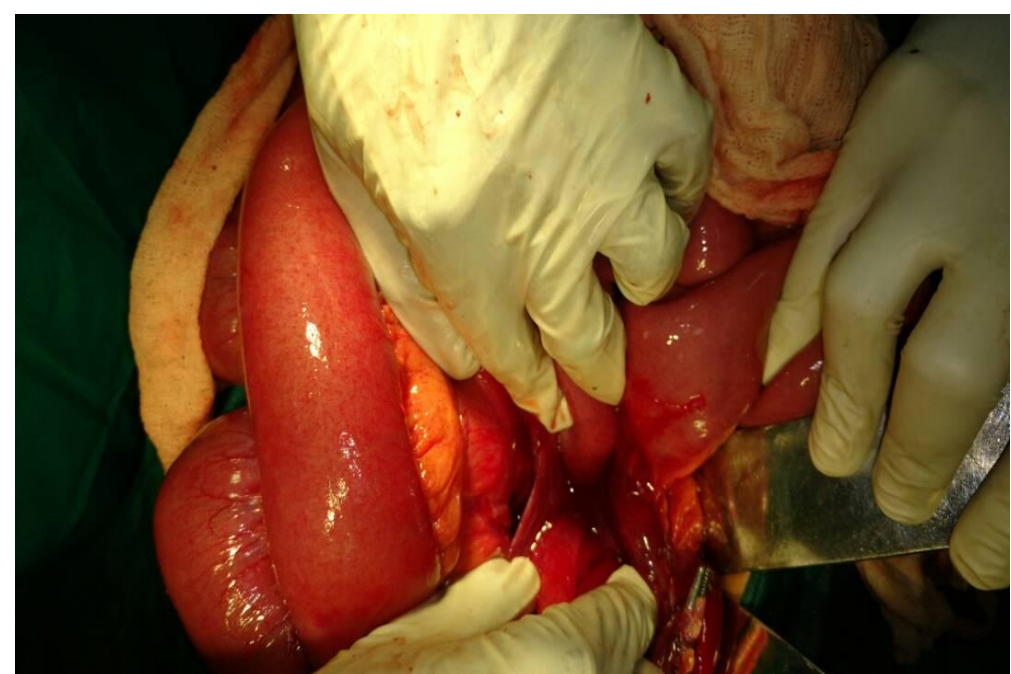

Fig 2: Peritoneal defect as seen after release of band and reduction of bowel loops.

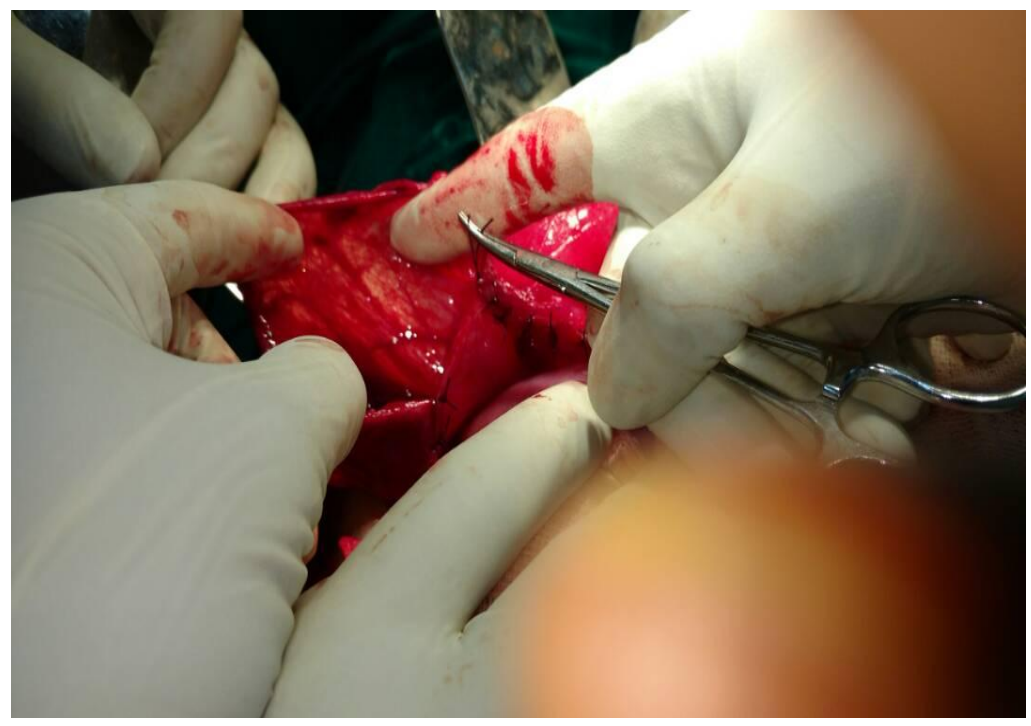

Fig 3: Obliteration of peritoneal sac. 


\section{Discussion}

Paraduodenal hernias have also been termed mesentericoparietal, congenital mesocolic, or retroperitoneal hernias. Treitz, who is credited with the first scientific description in 1857, described several peritoneal folds and fossae through which small bowel could potentially herniated. This type of hernia accounts for over half of all internal hernias ${ }^{3}$

Andrews proposed that paraduodenal hernia forms as a result of congenital anomaly in development of the peritoneum that arises during midgut rotation. As a consequence, small bowel becomes invaginated into an avascular segment of left mesocolon. The resulting small bowel thus becomes trapped between the posterior abdominal wall with the mesocolon and inferior mesenteric vein (IMV) forming the anterior wall of hernia sac. Peltier et al described the mechanism as either being acquired or related to anomalous rotation of midgut ( physiological herniation during the sixth and seventh weeks of gestation $).{ }^{4}$. All ages can be affected, with the mean age between $38-45$ years. ${ }^{4,5}$. A male prevalence has also been reported. ${ }^{3,5}$. A left PDH contains most of the small bowel, with the afferent limb being the fourth part of duodenum and efferent limb being the terminal ileum. After formation of the hernia, the colon usually retains its normal position. A right PDH originates from abnormalities arising during the second phase of embryonic intestinal rotation; it results in arrest of further ${ }^{7,9}$ rotation of prearterial segment of the gut in the right side of the abdomen. Continued rotation of postarterial segment leads to entrapment of small bowel behind the right colonic mesentery, with superior mesenteric artery forming the anterior edge of the hernia sac. Right paraduodenal hernias account for only $25 \%$ of all paraduodenal hernias and left sided hernias for the rest. ${ }^{2}$. These account for less than $1 \%$ of all small intestinal obstructions overall. ${ }^{6}$

With the total number of reported cases in literature being less than $500^{7}$, the natural history is to remain asymptomatic during the lifetime of a person. ${ }^{8}$ However, there is a $50 \%$ lifetime risk of obstruction when a paraduodenal hernia is present. Clinically, most patients present with recurrent episodes of abdominal pain, often progressing to partial or complete intestinal obstruction. ${ }^{9}$ Paraduodenal hernia presets as a non specific clinical picture, and often reduces spontaneously, leading to diagnosis difficulties ${ }^{10}$. Although a rare cause of intestinal obstruction, it has high mortality. On X ray, paraduodenal hernias were classically described as a clustering of small bowel loops in the upper right or left quadrants. Now, CT has become investigation of choice for diagnosing any type of internal hernia. With a significant life time risk of intestinal obstruction, elective repair is usually recommended. ${ }^{7,9}$.

Treatment of PDH follows the basic principles of hernia surgery, including reduction of hernia contents, resection of hernia sac, restoration of normal bowel anatomy, and repair of hernia defect. The small bowel may be manually reduced if the hernia orifice is large enough and the defect can be closed with non absorbable suture ${ }^{3}$. If the small bowel is oedematous, the hernia orifice is tight, or adhesions within the sac prevent manual reduction of the contents, the hernia orifice can be widened by excising the avascular plane to the right of IMV. Care should be taken to avoid damage to this structure and the left colic artery, both of which lie in close proximity to anterior edge of the orifice. If the orifice cannot be widened, the sac should be opened along the anterior wall and the contents reduced. Rarely, a decompressive enterotomy may be required. The sac should then be excised from the left of IMV toward the left colon taking care to preserve the marginal artery of Drummond. In cases of bowel strangulation and gangrene, the appropriate length of bowel should be resected and decision regarding anastomosis or an end ostomy should be made.

Recently, several authors have reported laparoscopic repair of right and left PDH. To date, 15 cases of laparoscopic PDH repair have been found in 11 articles in medical literature. Though in early stages, it can be expected that as surgeons become increasingly comfortable with laparoscopic techniques, PDH will be increasingly managed laparoscopically. Palanivelu et al ${ }^{7}$ reported on his experience with the laparoscopic approach. The operating time was 55-72 min. One patient had ileus of 3 days with spontaneous recovery. Hospital stay was in the range of 2-6 days. One patient had a recurrence, for which a laparoscopic mesh repair was successfully performed. Laparoscopic repair carries all the benefits of minimal access surgery, while providing a sound repair ${ }^{7}$.

\section{Conclusion}

Paraduodenal hernia is a rare congenital anomaly arising from an error of rotation of midgut. The duodenum and small intestine become trapped in a sac, lined by peritoneum, behind the mesentery of colon, either to the right or left of midline. This may be an incidental discovery at laparotomy or a rare cause of small bowel obstruction progressing to strangulation and perforation. Increasingly, the diagnosis is made on CT scan done for non specific abdominal pain or imaging for other reasons. PDH may be suspected on preoperative $\mathrm{X}$ ray examination which shows small bowel loops all on the right or to the left of midline. With a lifetime risk of obstruction being $50 \%$, surgery should be done even in uncomplicated cases, the approach being dictated by nature of the hernia. Careful consideration is required with respect to vasculature and sac contents. Obliterating the sac opening is essential. 


\section{References}

[1]. P.M. Amodio, A. Alberti, E. Bigonzoni, M. Piciollo, T. Fortunati, D. Alberti Left paraduodenal hernia: a case report and review of literature Chir Ital, 60 ( September- October (5)) ( 2008 ),pp.721-424

[2]. A. Blachar, M.P. Federle Internal hernia: an increasingly common cause of small bowel obstruction

[3]. Semin Ultrasound CT MRI, 23 ( 2002),pp.174-183

[4]. Jamal MK, DeMaria EJ: Internal hernia- congenital and acquired. In Yeo CJ, Dempsey DT, Klein AS,et al,editors: Schekelford surgery of the alimentary tract,ed 6. Philadelphia, 2007, Saunders, p 1120

[5]. Peltier J, Le Gars D, Page C, et al: The duodenal fossae: Anatomic study and clinical correlations. Surg Radiol Anat 27:303,2005

[6]. Antedomenico E, Singh NN, Zagorski SM et al: Laparoscopic repair of a right paraduodenal hernia. Surg Endosc 18:165,2004

[7]. C.H. Moon, M.H. Chung, K.M. Lin Diagnostic laparoscopy and laparoscopic repair of a left paraduodenal hernia can shorten hospital stay JSLS,10 ( January-March (1)) ( 2006 ),pp.90-93

[8]. C. Panalivelu, M. Rangrajan, P.A. Jategaonkar, N.V. anand, K. Senthilkumar Laparoscopic management of paraduodenal hernias: mesh and mesh less repairs. A report of four cases Hernia,12 ( December (6)) ( 2008 ),pp.649-653

[9]. R. Manji, G.L.Warnock Left paraduodenal hernia :an unusual cause of small bowel obstruction Can J Surg, 46 ( 2001),pp.455-456

[10]. R.A. Brigham, W.F. Fallon, J.R. Saunders, J.W. Harmon, J.C. d Avis Paraduodenal hernia: diagnosis and surgical management

[11]. Surgery, 96 ( 1984),pp.498-502

[12]. A. Cingi, P. Demirkalem, M.N. Manukyan, D.Tuney, C. Yegen Left-sided paraduodenal hernia:report of a case

[13]. Surg Today, 36 (7) ( 2006 ),pp.651-654

[14]. M.A. Khan, A.Y. Lo, D.M. Vande MAele Paraduodenal hernia, Am Surg,64 ( 1998),pp.1218-1222 